\title{
Foregrounding epistemology and everyday intuitions in a quantum physics course for nonscience majors
}

\author{
Michael C. Wittmann $\oplus^{1, *}$ and Jeffrey T. Morgan $\odot^{2, \dagger}$ \\ ${ }^{1}$ Department of Physics and Astronomy, University of Maine, Orono, Maine 04401, USA \\ ${ }^{2}$ Department of Physics, University of Northern Iowa, Cedar Falls, Iowa 50614, USA
}

(Received 8 July 2019; accepted 24 October 2019; published 4 December 2020)

\begin{abstract}
[This paper is part of the Focused Collection on Curriculum Development: Theory into Design.] In developing and modifying a course called Intuitive Quantum Physics for nonscience majors, several social and theoretical commitments informed our design decisions. We believed that the goal of a general education course should not be acquiring content knowledge alone, but more generally developing an approach to thinking scientifically. Thus, our course was designed to promote a deeper understanding of the nature of science through careful attention to students' personal epistemologies. We emphasized everyday situations, be they social activities or personal experiences, as analogies to be used during instruction. We used these everyday events to help students make sense of quantum physics, choosing the topic exactly because it seems otherwise counterintuitive. Through this work, we hoped to help students make connections between complex topics (in this case in science) and their everyday experiences.
\end{abstract}

DOI: 10.1103/PhysRevPhysEducRes.16.020159

\section{INTRODUCTION}

Developing a course in "modern physics" for students who have not studied advanced physics and mathematics presents challenges and opportunities that can be addressed using the results of the physics education research (PER) community. But, developing and teaching such a course can be problematic, as well. It is far too easy to answer the call to bring "the physics of the last century" to high school or general education students by having students memorize disconnected facts, watch computer simulations that they are unable to interpret, and leave with discouraging epistemologies and attitudes about the process of science. Rather than promoting their abilities as life-long learners, such courses stifle them. Our objective in creating a course called Intuitive Quantum Physics (IQP) was to create an effective course in quantum physics for nonscientists that avoided these pitfalls and strengthened our students' understanding of science. In this paper, we describe the design principles that guided the creation of the IQP.

The course was developed after the successful dissemination of a previous set of instructional materials, the New Model Course in Applied Quantum Physics [1], to

\footnotetext{
*mwittmann@maine.edu

jeff.morgan@uni.edu
}

Published by the American Physical Society under the terms of the Creative Commons Attribution 4.0 International license. Further distribution of this work must maintain attribution to the author(s) and the published article's title, journal citation, and DOI. hundreds of institutions around the world, and the publication of those materials as the Activity-Based Tutorials, Vol. 2: Modern Physics [2]. These materials had been developed for junior level engineers, a population that had introductory physics but not many of the other expected physics courses a major might have when taking quantum physics. While originally developing the New Model Course, we had not expected the engineering students to have many intuitions about quantum physics, but we found that there were a great number of intuitions about nonphysics phenomena that students used to make sense of the quantum world. This observation was the seed for our IQP course.

This paper is designed to go into ever increasing detail about our design process. In Sec. II, we describe the attitudes and positions that guided our work. In Sec. III, we begin with an overview of our course as it was originally envisioned, borrowing material from three previously published projects. We point out the shortcomings of our first implementation of the IQP course, and describe the ways in which an additional focus on personal epistemology transformed the course into its final form. In Sec. IV, we go into detail about the ways in which students used their personal intuitions to guide their thinking about two laboratory activities. The first set of intuitions helped students make sense of wave-particle duality. Students were able to reason about the physical phenomena in a way that mirrored the phenomena. The second moment of everyday knowledge was when students used their knowledge of driving a car to develop a method for solving the time-independent Schrödinger equation without using 
obvious mathematics. We end with a discussion of the ways in which course design depended on an ongoing feedback loop that helped us refine our guiding principles. Thus, this paper attempts to illustrate the ways in which the course was developed, but is obviously affected by the changes made along the way.

\section{MOTIVATIONS FOR OUR DESIGN}

In developing the Intuitive Quantum Physics course, multiple perspectives informed our decisions. We describe the broad principles behind our final design in this section. In the following section, we highlight the ways in which these choices affected our overall curriculum design and provide some detail about an important transition that led to the final version of our materials.

Our goals in the IQP course were to improve both student conceptual and epistemological knowledge. One objective was to improve student understanding of the fundamental ideas of wave physics and quantum physics, such as superposition, interference, wave-particle duality, quantization, and tunneling. At the time the course was being developed, research had shown that students typically had little experience describing the wave nature of the world [3-6] and even less experience making sense of the quantum world in which wave properties are applied to seemingly particlelike objects [7-9]. Another objective was to have students arrive at a view of science as the study of nature based on systematic descriptions of repeatable observations and logical reasoning. We had data from the Maryland Physics Expectations Survey [10] to show that many of the students taking our course viewed science as a collection of memorized facts. In general, we had found that in the context of quantum physics, students stated that ideas must be believed and not understood. We sought to change their epistemological framing of the topic. This led into the third objective of our course, that students could see that they themselves were capable of scientific reasoning. These students often made comments suggesting that they believed that science was done primarily by specialists. By teaching quantum physics, our project faced a special challenge in getting students to respond to this attitude when we discussed physics concepts not related to common observables.

One design challenge was to bring the excitement of modern science into the classroom. In modern science, one must acknowledge and work with its seemingly counterintuitive nature. Epistemologies (e.g., answers to the questions "how do you know?" and "why do you believe?" [11]) are of supreme importance when learning quantum physics. Should one simply believe in wave-particle duality, or can one reason about it by using observational evidence? Should one merely believe that particles can tunnel through potential barriers, or can one reason about tunneling using models? Quantum physics is an excellent topic in which to make explicit the epistemological content of scientific modeling. The IQP course was designed to bring together the ideas of quantum physics and the process of scientific modeling through explicit attention to useful epistemologies for learning.

At the time the IQP course was developed, all students at its land grant institution were required to take two semesters of science, one of which had to involve a laboratory. Any student attending the university had taken many science courses up to this point, from elementary through high school, but these students were not science majors. Several reasons could exist for not choosing a science major. These might include anxiety about mathematics or a dislike of science courses in general. They might also include a student needing to focus their attention elsewhere and not having time for more science classes in their program of study.

We wished to design a course that would appeal to all these students, especially since this was the last science course that many would take. We wanted to leave these students with a good last impression of what science could be. We chose to emphasize the nature of scientific thinking in addition to the particular science content. We wanted to challenge them and appeal to people's curiosity about a topic that seems to make no sense, but is obviously an area of scientific knowledge: quantum physics. Students in the course were aware of counterintuitive ideas like waveparticle duality, and we wished to explore these ideas with them in more detail. Further, students are often excited by and curious about these ideas, and we hoped to appeal to this sense of curiosity.

We believed that reasoning through epistemologically difficult topics is something that should be available to all students, not just physics students in the later years of their major. To address the counterintuitive nature of many quantum physics ideas, we chose to emphasize three issues of epistemology: how do you know something, why do you believe it, and how can you explain it. We used these questions to guide students to model systems that cannot be seen, explore contradictions even when the contradiction cannot be resolved, and ask precise questions and make predictions of even counterintuitive situations. In Sec. III, we will describe how we made the answers to these three questions explicit in the course. In Sec. IV, we will provide two examples from different stages of the course to illustrate specific answers to these three questions of epistemology.

Because the course was not based on canonical mathematical problems in quantum physics, we were free to think differently about how we modeled systems. A typical quantum physics course will rely heavily on the mathematics as its epistemological frame, in that the implicit answer to the question "how do you know this?" is "because the mathematics told me so." In our course, in contrast, mathematics would be used, but would not supplant other forms of reasoning. More subtly, the 
mathematics would be packaged in a form where students would not think that they were solving a difficult mathematics problem.

We rewrote the Schrödinger equation into a form that could be understood graphically and intuitively. We reinterpreted boundary conditions and normalization as a set of rules about drawing wave functions. Using this toolbox, we were able to interpret finite square well systems, model a kind of molecular bonding by discussing the lowering of ground state energies for a system of two adjacent finite square wells, discuss spectroscopy and therefore astronomical measurements such as the discovery of helium in spectra taken from the sun, and discuss alpha decay and STM measurements by applying our ideas to quantum tunneling. In Sec. IV B, we will discuss the graphical interpretation of the wave function, and how we tapped into student knowledge about driving down a road to help make sense of the "graphical Schrödinger equation."

Because of our strongly constructivist stance toward student learning [12], essential topics of the physics were not introduced during lecture time. We assumed that our students would be bright and engaged with the ideas, if presented in an engaging manner, and that they could bring many ideas to the classroom in a way that could be leveraged for instruction. Thus, the largest part of the course involved students working in small group activities to build new concepts and create new knowledge, while the large-lecture part of the course was built around discussion and debate of ideas within a Peer Instruction format [13], rather than presentation of ideas by the instructor. Students had $3 \mathrm{~h}$ laboratories in which they worked in small groups on tutorial-like activities that helped them develop their knowledge. We avoided common historical or mathematical activities, and rarely engaged in typical modern physics experiments. Instead, we methodically built a toolbox that included wave physics ideas of superposition and interference, classical ideas of probability and probability density, an exploration of the idea of potential energy, and an interpretation of the wave function. Because working without interruption in small groups for many hours can be boring and frustrating, we used regular "whiteboard meetings" [14] to give students a chance to present their work to the larger class and engage with each other's ideas. Because we wanted students to develop ideas slowly and carefully rather than being told ideas by the instructor, lecture time was spent addressing dilemmas that students had, answering questions, and fostering a debate among students about the concepts they had learned during the lab period. Lecture time was also used for explicit discussion of the three epistemological questions described above, as well as explicit engagement with ideas of conceptual coherence and consistency, even in counterintuitive topics such as wave-particle duality.

In sum, our design principles were guided by choices about epistemology, physics content, the use of mathematics
TABLE I. Summary of design principles that guided our work. Our decisions were guided in large part by the student population of nonscience majors, with many taking their last academic science class of their careers.

\section{Design principles}

Using quantum physics as a platform to study epistemology

Aligning instructional ways of reasoning with student abilities in qualitative, conceptual, and graphical reasoning

Tapping into student intuitions about everyday life to find examples of the reasoning used in quantum physics

Using small-group activities in multihour labs to allow all students the time needed for learning difficult ideas

in physics, our knowledge of the incoming student population, and our principled stance toward constructivist learning (see Table I). We chose to focus on epistemological questions which we believed could be investigated in detail using quantum physics. We chose to avoid an overly mathematical approach which might turn students away. We engaged in qualitative and conceptual reasoning, often using graphical models to help us draw conclusions about the systems we investigated. We recognized that students would have many intuitions that would help them make sense of the models of quantum physics that we were building, and designed course materials to tap into these intuitions. Finally, we chose to provide students with ample time for working in small groups to construct their own understanding of the physics, minimizing the use of lecture as a vehicle for introducing new ideas.

\section{BORROWED COURSE MATERIALS AND A FOCUS ON EPISTEMOLOGY}

In this section, we describe the ways in which the design principles described in the previous section guided our adoption and modification of existing materials. We give two more explicit examples in the next section.

\section{A. Use of existing materials}

The IQP was originally designed to use modified materials from three published sources. We adapted the following tutorials from their original design of small-group guided inquiry worksheets taught in discussion sections of a large lecture course: The Activity-Based Tutorials, Vol. 1 [15,16] about wave propagation and superposition, the Tutorials in Introductory Physics [17] about optics and wave interference, and the New Model Course in Applied Quantum Physics [1], which was published as the Activity-Based Tutorials, Vol. 2 [2]. Each of these came with an extensive research basis that was well documented in the literature [3,5,7,18-21]. In addition, new materials were created, for example in the areas of teaching energy, probability, and probability density [22].

In choosing tutorials, we had to address a design inconsistency. As part of their design, tutorial-style materials were 
developed to be used in small-group learning classrooms in conjunction with lectures in which the majority of content would be introduced. Typically, they replaced problemsolving recitations. Stated differently, the assumption was that students would have been introduced to the important concepts before doing the tutorial. In contrast, in our quantum physics course for nonscience majors, content was introduced primarily during the weekly laboratory periods. We tried to keep the materials reasonably faithful to the intentions of the developers even as we used them in ways not originally intended.

This often required that we provided students with more examples and observations about the physics, where possible. For example, students were introduced to wave propagation and superposition using materials from the Activity-Based Tutorials [15]. They observed waves on a spring but also observed waves in large water ripple tanks that we made for the course. Students could then observe interference patterns in the water, model them using the ideas of superposition, and discuss nodal lines where the water does not move, consistent with handouts that were part of the Tutorials in Introductory Physics. Importantly, they were asked to make parallels between dark spots on a screen during light interference and nodal points in the water. Thus, we helped students build a conceptual model of the wave nature of light by making analogies between the patterns seen in the interference of nonobservable waves (light) and observable waves (on a string or on water). In Sec. IV A, we provide more detail about how this analogy was made.

The different student audience was also of some concern to us. Tutorials were developed for courses typically taken by physics majors and engineering students with some background in calculus and at least some level of comfort in the mathematics used in such courses. We changed the level of mathematics that was used, typically by splitting up aspects of quantitative reasoning into smaller steps for students to work through. We also changed some of the mathematical work into work of graphical interpretation (as discussed in Sec. IV B). We added excerpts of other tutorials that were necessary for students to develop background knowledge for a particular activity. Sometimes, we developed new materials based on existing materials, such as when we helped students develop an understanding of probabilities and probability density [22], using ideas first introduced by Bao et al. [9]. Other times, we developed entirely new materials for students, such as when we developed the idea of potential energy diagrams by tapping into knowledge about conservation of energy and the idealworld situation of mechanical energy being conserved (e.g., there are no thermal dissipative effects).

\section{B. A focus on personal epistemology}

Even with these modifications, there were problems with our course. First, we observed students losing interest in the material as it became less and less connected to their everyday experiences. Using tests, ungraded quizzes, and specially designed surveys to evaluate student learning, we could show that students were learning difficult concepts while having little previous background in physics and likely none in the content of the course. But, they were not developing skills consistent with our design principles. The problem was that the tutorial materials we used contained very little explicit discussion of epistemological issues. Students were rarely asked, "Why do you believe this?" When asked to explain their reasoning, students were typically being asked to explain the logical steps used to arrive at an answer. They were not being asked to evaluate why they believed the outcome of this reasoning, and were only rarely asked to consider or evaluate alternative responses. Such thinking is of great importance when learning counterintuitive ideas in quantum physics. In our response to this issue, we were greatly influenced by the University of Maryland project on Learning How to Learn [23], with its emphasis on developing personal epistemologies [11,24-26]. We were thus influenced by the materials later published as the Open Source Tutorials [27,28], which we knew of while they were in development.

The influence of the Learning How to Learn project could be seen in the language we eventually developed for our materials. We tried to use a language that was more open about the learning process with students. Also, we were more explicit as we tried to help students tap into their everyday intuitions by using language more consistent with their everyday talk than with formal physics questions. This required adding new questions to the existing tutorial materials, or modifying them to use a more colloquial language. By connecting to their everyday language and examples from everyday life, we wanted to promote the idea that the science they were learning was accessible to them. We provide an example when discussing waveparticle duality in Sec. IV A. Sometimes, our changes were more than just linguistic. When helping students find the functions that are solutions to the time-independent Schrödinger equation, we designed the activity around common tasks such as driving that are familiar to students. This example is described in detail in Sec. IV B.

\section{Examples of revisions based on observation, evaluation, and discussion}

In the IQP, we modified existing published materials to better match our course structure and student body. We then modified the materials to include a focus on personal epistemology as a way of strengthening students' attitudes toward and understanding of the nature of science. In teaching the course, several observations and evaluations led to further changes. We provide two examples out of many.

One of our design principles was that students engage in small-group learning with ample time to allow all students the time needed for learning difficult ideas. We observed, 
though, that having the course move at a different pace for each student group placed each group in isolation from each other and put a large burden on the single teaching assistant in a room with many groups of students working on different topics. The lab assistant was unable to spend enough time with students, which meant that students often moved forward with activities when they had not adequately discussed ideas in the worksheets with an instructor who could ask for more detail about their thinking. Without adequate facilitation, students often used incorrect ideas as they answered further questions. Once corrected, they would complain about having spent pages doing the wrong thing, and many lost confidence in their abilities and became frustrated with the material. We addressed these concerns by trying to have groups work at close to the same pace, and then having full-class whiteboard meetings in which students would present their reasoning and thinking about the work they had just been doing [14]. By having students present to the class, we found that students were less frustrated, and there was more engagement with ideas.

A second change was also based on our observations of students in the laboratory periods. Often, students needed more space to write out their reasoning than was provided in the original worksheet handouts. We modified the documents to give this space, and found that it suggested to the students that they write more and provide more information. They would write to fill the space that was given, providing more opportunity to develop and make public their reasoning about a particular situation. At the same time, we redesigned some parts of the activities to prevent that information from being given. Sometimes, we replaced a long series of questions about different cases or scenarios in a situation with a table that summarized the results of these questions. Students only had to fill in the table. By doing so, they engaged verbally in reasoning about the physics, but were prevented (by the format of the document they were using) from writing much about it. In both these cases, the layout and design of the document was used to influence student work. We provide examples of such tables below.

Many other changes were made in the course and are not reported here. For example, we used data from the MPEX (both version 1 [10] and version 2 [29]) asked before, during, and at the end of the semester to investigate when students' attitudes and expectations about the coherence and consistency of the physics changed. Results indicated areas of improvement and led to a redesign of the course to focus more on personal epistemology, as discussed above.

\section{Where we arrived}

Our modifications of existing tutorials and creation of new materials as well as our design of the instructional setting were driven by our design principles. First, we believed that all students in the course could succeed and we built a laboratory environment in which students were given many hours to complete their work. They could determine their own pace, and they were given responsibility for their learning. But, when we observed problems with this approach, we modified instruction to have white board meetings that allowed students to interact with each other's ideas more. Second, we believed that students could carefully construct their own knowledge by using welldesigned materials, and that students would respond to challenging work that was at the appropriate level of difficulty. For example, we simplified the mathematics and quantitative reasoning that students used to arrive at their responses. We also included more elements from their everyday lives, both in the language of the lab activities and the examples we used to develop the ideas of quantum physics. Finally, we worked out a progression of ideas that let us discuss personal epistemology as a vehicle for learning quantum physics.

We summarize the large-scale structure of the design of our materials in Fig. 1. The figure represents how all of our foundational instructional materials had to pass through a filter in which we thought about how to include issues of personal epistemology in instruction. Further, because the course was taught for several years, we were able to use an evaluation process that created a feedback loop of observation, group discussion among the research team, and course revision.

In Table I, we summarized our design principles. In Table II, we summarize how these affected our course design and revisions. In sum, we asked students to bring their knowledge and experience into the classroom, we asked questions to tap into personal epistemology, we tried to make use of everyday intuitions, and we developed more ways of creating collaborative, student-centered instruction.

The end result of our work was a course containing the elements shown in Table III. There were 3 units to the

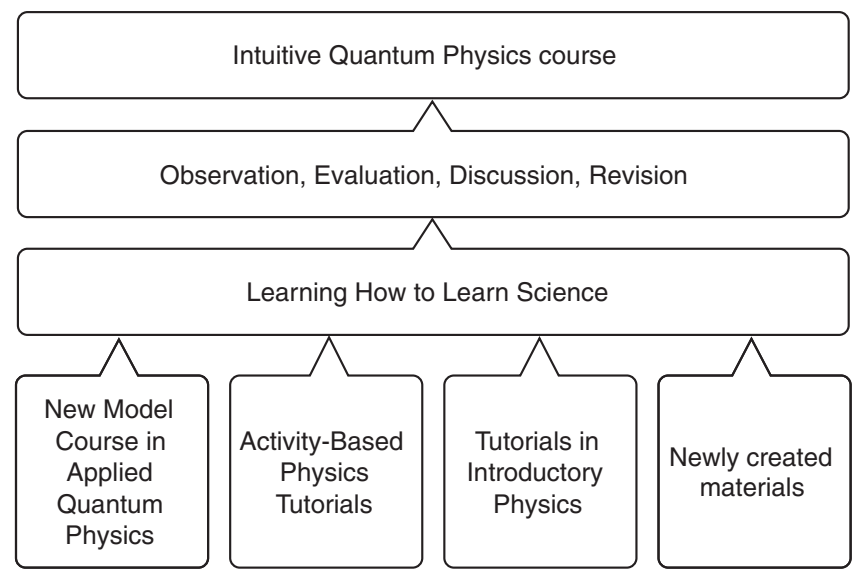

FIG. 1. The Intuitive Quantum Physics course was built on a foundation of research-based materials modified to be more consistent with work done on developing personal epistemologies in the classroom. 
TABLE II. How materials were adapted, consistent with our design principles.

\begin{tabular}{|c|c|}
\hline Design principles & $\begin{array}{l}\text { Enactment of design principles } \\
\text { in course }\end{array}$ \\
\hline $\begin{array}{l}\text { Epistemology as a vehicle } \\
\text { for understanding } \\
\text { quantum physics }\end{array}$ & $\begin{array}{l}\text { Adapting tutorials to include more } \\
\text { explicit discussion of questions } \\
\text { of personal epistemology }\end{array}$ \\
\hline $\begin{array}{l}\text { Student understanding of } \\
\text { the nature of reasoning }\end{array}$ & $\begin{array}{l}\text { Modifying tutorials so that } \\
\text { reasoning happens in smaller } \\
\text { steps, or uses graphical rather } \\
\text { than mathematical reasoning. }\end{array}$ \\
\hline $\begin{array}{l}\text { Tapping into everyday } \\
\text { intuitions }\end{array}$ & $\begin{array}{l}\text { Using everyday language and } \\
\text { examples from everyday life } \\
\text { with reasoning parallel to } \\
\text { physics. }\end{array}$ \\
\hline $\begin{array}{l}\text { Student-centered } \\
\text { instruction }\end{array}$ & $\begin{array}{l}\text { Providing students with a context } \\
\text { for large-group discussion that } \\
\text { encourages deeper exploration } \\
\text { of ideas and more }\end{array}$ \\
\hline
\end{tabular}

course. Unit 1, on waves and optics, built up necessary ideas about superposition, interference, and the observation of these phenomena in the world. Unit 2, on energy, probability, and the wave equation, was focused on toolbox development that would lead to a model that allowed for further exploration of many situations. Unit 3, on quantum physics, applied the toolbox to spectroscopy, molecular bonding, and radioactive decay. Where a typical syllabus of a course might only have listed the content topics ("What do you know?"), we also included information on epistemology ("How do you know?").

Each unit had some touchstone examples to which we returned repeatedly. In Unit 1, these were typical examples from wave physics. In Unit 2, one touchstone example was of a cart bouncing between two walls. We used its potential energy diagram to develop the idea of a finite square well.
We used the probability of being found in various regions in the well to develop the idea of probability density. A second touchstone example, of driving down a graph of the wave function as if driving down a road on a map, will be discussed in greater detail in Sec. IV B. In Unit 3, the touchstone example was the quantized energies of a finite square well, and understanding these energies to make sense of spectroscopy, building a model of molecular bonding, or understanding quantum tunneling and building a model of alpha decay.

Finally, each unit ended with a point of contention or a dilemma. Unit 1 ended with the discussion of wave-particle duality, which suggested that we needed to learn more about probability and energy in order to understand electrons. Unit 2 ended with using the Schrödinger equation to find the probabilities of locating an object in a given place, as will be discussed below. Unit 3 ended with explicit discussion (during lecture) of the nature of scientific reasoning and the broad applicability of models to many different situations as an example of the general value of the model.

\section{ILLUSTRATING THE COURSE DESIGN}

Having described our design principles in Sec. II and the overall course structure in Sec. III, we now turn to two specific examples of how our design principles were expressed in the course.

\section{A. Analogies and dilemmas with waves and particles}

Unit 1 ended with observations of images taken when monochromatic light or electrons are incident on appropriately narrow double slits at very low intensity. In such a situation, images such as the one in Fig. 2 are created, where dots are clearly visible, yet the effects of interference are also shown [30].

In the IQP, students used similar figures (see Fig. 3) to discuss the probability of finding an electron incident in a

TABLE III. Intuitive Quantum Physics units. In each, we describe the content, guiding epistemological frame, touchstone examples, and physical dilemmas that guide instruction.

\begin{tabular}{|c|c|c|c|}
\hline Unit & 1. Waves and optics & 2. Toolbox building & $\begin{array}{l}\text { 3. Applying tools to quantum } \\
\text { physics }\end{array}$ \\
\hline $\begin{array}{l}\text { Content topics: "What do } \\
\text { you know?" }\end{array}$ & $\begin{array}{l}\text { Propagation; superposition; two } \\
\text { source interference; light as a } \\
\text { wave }\end{array}$ & $\begin{array}{l}\text { Energy; probability; wave } \\
\text { function; Schrödinger equation }\end{array}$ & $\begin{array}{l}\text { Quantization and discrete energy } \\
\text { levels; bound states; quantum } \\
\text { tunneling }\end{array}$ \\
\hline $\begin{array}{l}\text { Epistemology: "How do } \\
\text { you know?" }\end{array}$ & $\begin{array}{l}\text { Reasoning based on direct } \\
\text { observations; trusting intuitions }\end{array}$ & $\begin{array}{l}\text { Building from intuitions and } \\
\text { common sense; trusting logic }\end{array}$ & $\begin{array}{l}\text { Refining and combining intuitions; } \\
\text { evaluating counterintuitive ideas }\end{array}$ \\
\hline Touchstone examples: & $\begin{array}{l}\text { Waves on a spring; water waves; } \\
\text { bulbs and masks; two slit } \\
\text { interference }\end{array}$ & $\begin{array}{l}\text { Cart between two walls; "driving } \\
\text { down a function" to describe } \\
\text { continuity and curvature }\end{array}$ & $\begin{array}{l}\text { Finite square wells; spectroscopy; } \\
\text { alpha decay and tunneling }\end{array}$ \\
\hline End-of-unit issue: & $\begin{array}{l}\text { Wave-particle duality for photons } \\
\text { and electrons }\end{array}$ & $\begin{array}{l}\text { Using an equation to define the } \\
\text { probability of locating an object }\end{array}$ & $\begin{array}{l}\text { Broadly applicable models as } \\
\text { evidence of a good model }\end{array}$ \\
\hline
\end{tabular}



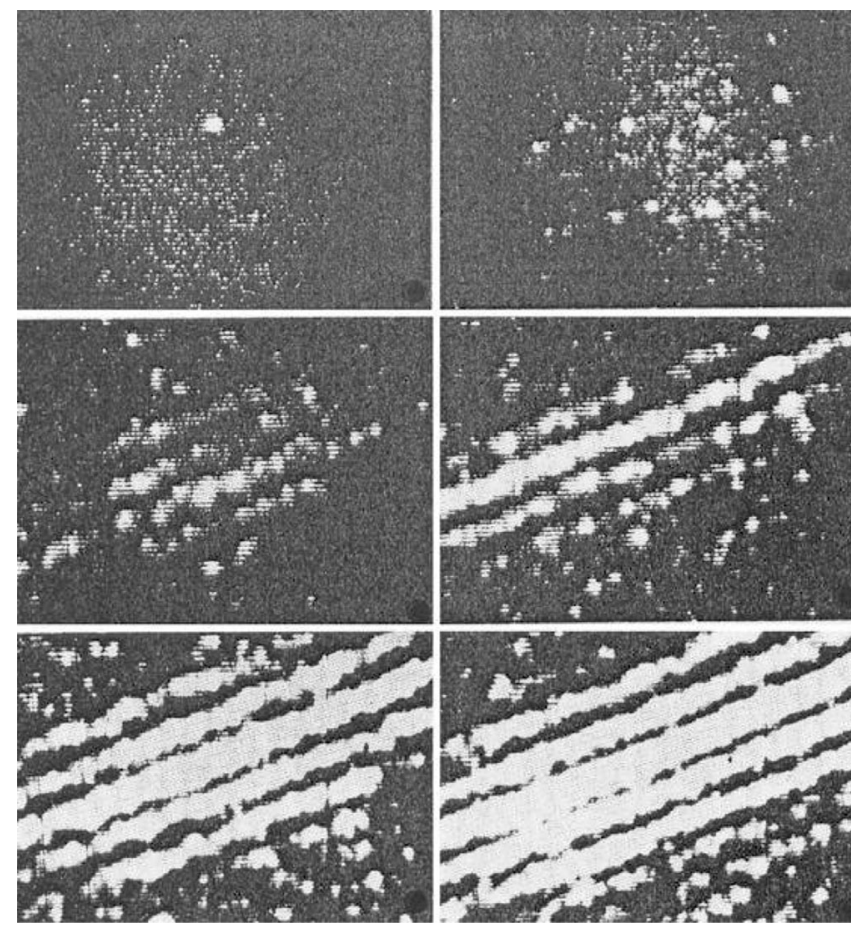

FIG. 2. Results from a low intensity two-slit electron interference experiment [30].

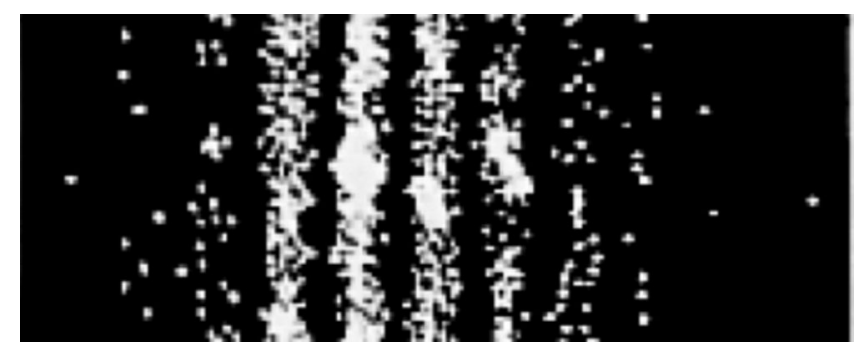

FIG. 3. Results from a low intensity two-slit electron interference experiment [31].

given region of space (e.g., highly unlikely to be incident on a dark band or close to it, most likely to be incident on the screen at the middle of a bright band, and so on). The need to understand the concepts of wave-particle duality, probability, and the energy of electrons incident on the double slit led to the second unit of the course. In this section, we discuss not this transition in content from waves to energy and probability but the ways in which we helped students make sense of the dilemmas posed by waveparticle duality. This analysis illustrates how our design principles affected the curriculum design and instruction.

\section{Consensus knowledge}

During unit 1, the answers to questions about epistemology arose naturally out of the study of the content. We know something because we observe it happening - light seems to come from sources, images are locations our eye imagines those sources to be, and so on. We believe that a thing happened because we saw it happen-waves interfere and we observe that there are places (with the name nodal lines) where it looks like nothing is happening, and we can explain how two waves coming together lead to that result. We can then explain light waves (which we cannot see) by comparing them to water waves, which we have experienced in real life (and not just in lab). These epistemological responses were unproblematic until they required the observations shown in Fig. 2, where electrons (which students thought of as particles) showed wave behavior, even when arriving on a screen as individual dots. To illustrate how our design principles were applied to this activity, we discuss the language we used with students, the logic that they developed, and the observations we made to show that students had developed a deep understanding of wave-particle duality.

We begin by discussing how we talked about knowledge building in class. When making the analogy between two-slit water wave interference and light interference, we introduced a white board meeting by asking students to think about the effect of perfect two-slit interference with water waves, where the water was dyed and the interference pattern was therefore "permanently drawn" onto a gray screen:

"As we just said, this gray barrier model is very important, and we want to take time to arrive at a class-wide consensus on it. On your whiteboard, copy the four gray barrier sketches you made on the previous page. (If you haven't come to a group consensus with your tablemates, now would be a good time to do so!)

"We'll have a class discussion about the gray barrier model. After the class comes to a consensus, use the diagrams below (the questions are identical to the previous page) to record the ideas developed, especially if they differ from what you predicted on the previous page."

This excerpt is typical of the language used in the course around building consensus knowledge, shared by all. We told students that the example was important and that we needed to make sure that everyone in the class arrived at a consensus about what was observed. We noted that this consensus making began within a group, and that students would then have a chance to record the consensus idea individually. We left open the possibility that there might be differences in what was originally answered and the ideas that the class developed, implicitly showing that it was okay to disagree and that the process of building consensus knowledge might require changing one's mind about something. And, we did it all in a friendly, relaxed language. We note that these positions were strongly influenced by work done by Hammer $[25,32,33]$, especially the idea of leaving open disagreements about content until resolved through classroom discussion. 


\section{Coherence and consistency of knowledge}

The same relaxed language was used to help students think about real-world examples of events as well as to remind them of previous work that they had done. For example, we presented students with the following series of questions when asking them to think about the differences between waves and particles passing through two slits.

1. Imagine shooting a paint ball at a wall (for those who don't know, a paint ball is like a little balloon filled with paint). What would you see?

2. Imagine randomly shooting paint balls at a wall with two holes in it. What would happen? What would we see beyond the wall if we had a screen there?

3. Imagine a dyed water wave hitting a gray wall. What do you see on the wall? Is one place different from another? (If you haven't already done so, think about last week's gray barrier.)

4. Imagine another dyed water wave coming up on a wall with two slits in it. What happens? What would you see on the wall behind? (Yes, we've studied this idea in previous tutorials - be brief in your summary!)

We used paint balls because we wanted high speed projectiles that traveled in as straight a line as possible and left behind evidence of where they landed; we did not want to use bullets as our example. We left open the (very likely) possibility that many did not know what paint balls were, but described them in the simplest possible language. In other discussions, we used wet tennis balls, which would leave behind a mark where they landed. We created contrasts between particles (in this case paint balls) and waves passing through double slits. In question 3 , we reminded them of their past work on dyed water and gray barriers, and in question 4, we reminded them of the board meeting from the previous week. From these and many other similar questions, we promoted the idea that the science the students were learning was also coherent and consistent.

\section{Personal epistemology in understanding dilemmas}

Students were given a series of individual images that led to the image shown in Fig. 3 [31], much like the images shown in Fig. 2. To make sense of these images, students answered a series of questions which made several analogies to their past work. These built on the consensus knowledge developed in class and emphasized the coherence and consistency of our knowledge of the world.

Students observed that bright bands (where there are many dots on top of each other) are separated by dark regions where there are no dots incident on the screen. The dark regions between bands were consistent with observations of light interference from lasers and descriptions of water wave interference from two sources separated by a few wavelengths. The dots on the screen were consistent with observations of things like paint balls or wet tennis balls hitting a wall (or any particlelike object hitting a screen). Knowing that the electrons had passed through two narrow slits led to two difficult statements. Perhaps electrons were a single particlelike thing, which we conclude because we observed them by seeing one dot on a screen. These particles went through two slits at the same time, otherwise, interference would be impossible. Problematically, particles shouldn't go through both slits. In contrast, perhaps electrons were wavelike things, which we conclude because we observed an interference pattern. These waves arrive on the screen as individual dots and the pattern is only discernable after some time has passed. Problematically, waves should not arrive in a single location but show up everywhere on a screen. (The answer to question 3, above, was a precursor to this observation, with ink on the whole gray barrier, and no nodes.)

During lecture-based discussion, students had arrived at different ways of dealing with this situation. Perhaps this was an appropriate time to "choose one," and a thing would be either particle or wave. This was similar to driving from one location to the next, in that you could not take both a highway and the back roads at the same time. In contrast, perhaps this was an appropriate time to "do both," and a thing could be both a particle and a wave. This was similar to wanting ketchup and mustard on a hot dog. The waveparticle duality observations (such as those provided in Refs. [30,31]) were more complicated than this and required both "choose one" and "do both" reasoning about the same situation, as we now discuss.

As part of our evaluations, we asked examination questions that required students not just to use multiple modes of thinking but also to be aware of their use of multiple models. In one examination, we gave students the image shown in Fig. 3 and asked them to describe what specific element showed evidence of the wave nature of electrons (correct answer: the interference pattern) and what specific element showed evidence of the particle nature of electrons (correct answer: the dots). Students answered these questions very well. Then, reaching back to language that had arisen in class, they were asked "How is this an example of both a 'do both' and a 'choose one' situation?" A correct answer would use the reasoning describe above, namely that both wave- and particlelike behaviors are seen, but you have to choose only one when describing certain elements of the observation. Though students had discussed many aspects of the interference pattern, they had not previously engaged in this particular conversation. Nearly $3 / 4$ of the students $(74 \%)$ gave an answer we believed was satisfactory. As one student stated,

"In this you have to choose at which points or parts the electron is acting as a wave and when it is acting as a particle. So like I said [previously], I chose that the electron is acting like a wave while going through the two slits but is acting as a particle when it hits the screen." 
For the student, the electron was both a wave and a particle, but for some observations one had to choose only one property to describe it, not both.

These and other results suggested that students were able to discuss general ideas of choice and coherence in the context of the central counterintuitive dilemmas of quantum physics. In addition, they were able to apply the ideas of wave physics, including superposition and interference, and apply them by analogy to things that students had previously not considered to be waves.

\section{B. The graphical Schrödinger equation}

In the second unit, we introduced concepts of probability, energy, and interpreting graphs to help students make sense of quantum physics through the time-independent Schrödinger equation. Concepts of probability were introduced with discrete systems (such as coins or dice) and extended to macroscopic physical systems. Students described the probability density of a ball thrown in the air based on how likely it was to be observed in the top, middle, or bottom third of its trajectory $[9,22,34]$. Concepts of energy were introduced using common sense intuitions about kinetic energy. Students discussed a dropped ball that bounces perfectly elastically, a cart moving up and down an incline plane, a cart bouncing perfectly elastically between two hard walls, and oscillating masses hanging from a spring. In each case, they used their intuitive sense of the conservation of energy in ideal, loss-less systems and qualitative reasoning about the kinetic energy to arrive at a graphical model of potential energy. Students were asked to distinguish between the energy of a system (e.g., the cart between two walls) and the energy of an object within the system (the cart itself). Later, students discussed the potential energy of a quantum mechanical system and the energy of the electron in that system.

\section{Rethinking the Schrödinger equation}

A major leap occurred with the introduction of the time-independent Schrödinger equation (1) to students. We simply asserted that the equation itself was a law of nature that described electrons and other tiny objects, which is mostly no different from how it is introduced in a typical physics course. But, we did not present it to them in the form that a physicist would be familiar with. Instead, we took the time-independent Schrödinger equation

$$
-\frac{\hbar^{2}}{2 m} \frac{d^{2} \psi}{d x^{2}}+U \psi=E \psi
$$

and rewrote it to separate the differential term from the functional terms:

$$
\frac{d^{2} \psi}{d x^{2}}=-\frac{2 m}{\hbar^{2}}(E-U) \psi
$$

Since students have little to no experience with calculus (and we expected no experience with differential equations), the differential term was reformulated in graphical terms, while the other terms in the equation were also redefined:

$$
\text { Curviness of } \psi=-k\left(\mathrm{TE}_{\text {particle }}-\mathrm{PE}_{\text {system }}\right) \psi \text {. }
$$

Certain constants have been buried in a new constant $k$, while others have been rephrased to be consistent with the previously developed descriptions of energies and potential energy graphs.

In addition, we asserted that there were rules that had to be followed when trying to draw a wave function. First, the area under the curve had to be finite. This was our way of asserting that the wave function had to be normalizable. Second, in transitions from one region of space to another where, for example, the wave function might change from sinusoidal to exponential form, the transition had to be smooth: no kinks in the line. This was our way of asserting continuity in the function and its first derivative. The graphical Schrödinger equation and these two rules were enough to help us start unit 3 . In this section, we describe how we found solutions to the graphical Schrödinger equation and the rules about normalizability and continuity to arrive at quantized energy levels.

We used a graphical solutions method which we call a "guess-and-consider-the-consequences" way of solving Eq. (3) [35]. To solve the equation, students assumed $\Psi$ was positive in a region where the total energy of a particle was larger than the potential energy of a system (say, inside a finite square well). Using Eq. (3), students would conclude that the curviness of $\Psi$ was negative. Similarly, if $\Psi$ was negative, the curviness was positive. Curviness was interpreted as the amount by which one turned a steering wheel while driving down the function as if driving down a road on a map. So, if students imagined crossing the axis at some angle, they would have to turn the steering wheel more, the further they were from the axis. At some point, they would have turned back toward the axis, and would have to straighten the wheel until they were pointing straight again, at just the moment that they crossed the axis. The process would repeat, except the steering wheel would turn the other direction. (We assumed that students knew that turning a wheel led to a car turning more, even if they had not driven before and had perhaps only seen people drive in, for example, movies, and we never encountered a student who did not understand this idea.) In describing this motion, they would have defined a sinusoidal curve. In the graphical Schrödinger equation (3), the amount of curviness (with all other terms constant) is determined by the value of the wave 
Ed takes a driving course in which he has to drive his car around pylons as shown below

as Rand watches him. He drives at a constant speed along the curvy path.

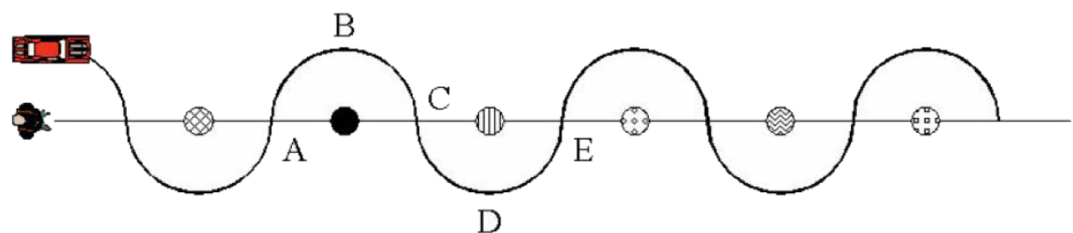

Ed's steering wheel has a bar that points straight up when the wheel is not turned.

In the table below, record what the nearest pylon is, whether Ed is to the left or

right of the pylon according to Rand and the center line, and draw a picture of the

orientation of Ed's steering wheel for each part of the path. We've filled in the first row

for you.

\begin{tabular}{|l|l|l|l|}
\hline & Nearest Pylon? & $\begin{array}{l}\text { Left or Right of } \\
\text { Pylon? }\end{array}$ & $\begin{array}{l}\text { Steering Wheel } \\
\text { Drawing }\end{array}$ \\
\hline A to B & Solid & Left & \\
\hline $\begin{array}{l}\text { B to C } \\
\text { (etc.) }\end{array}$ & & & \\
\hline
\end{tabular}

At each of points A, B, C, D, and E, is his steering wheel turned? If so, which way?

Explain how you can tell.

FIG. 4. Excerpt 1 from the curviness tutorial. Students begin by thinking about driving when holding a steering wheel steady, and fill out a table that collects multiple pieces of information.

function. ${ }^{1}$ Students also explored the situation in which the total energy of the particle was less than the potential energy of the system. In such a situation, one turns the wheel away from the axis, the further one drives away. This situation is akin to exponential growth (though in real life, one could imagine turning around and driving backwards, which does not happen in exponential growth).

\section{Using everyday events: Driving down a function}

In developing the meaning of curviness when thinking about the graphical Schrödinger equation, we had students consider "Ed" who was driving, and "Rand" who was standing so that he looked down the horizontal axis (from the origin) as Ed drove back and forth (when driving in

\footnotetext{
${ }^{1}$ In another situation with a similar differential equation, the amount of force of a spring (and acceleration of an attached object) is determined by the displacement of the object from the spring's equilibrium. In our situation, we defined the second derivative as "curviness," without further physical meaning.
}

half-circles, as shown in Fig. 4). In class, we pointed out that Ed was based on a real person, a former race car driver who had been a member of our University research group. This was our semiserious way of introducing students to the idea of driving as a valid way of making sense of the shapes of equations. We present some excerpts from the lab activity to illustrate the progression of ideas.

First, students were asked to think about Ed driving with his steering wheel held fixed (see Fig. 4). As the tutorial progressed, students were able to use a series of transparencies with different sized circles to determine the "curviness" at a given point by considering which circle best matched the curve of the graph at that location. This is akin to drawing the best fit tangent line at a given point, but for the second derivative. We used the idea of how far one would turn the steering wheel to drive in that circle, with the tightest turn being the "most curvy" and the least amount of turn being the least.

An important aspect of the curviness activity was to collect information in a way that helped students observe 


\begin{tabular}{|c|c|c|c|c|c|}
\hline \multicolumn{5}{|c|}{ s-function } \\
\hline $\begin{array}{c}\text { When } \\
\mathrm{x}=\end{array}$ & $\begin{array}{c}\text { Value of function } \\
+ \text { or }-\end{array}$ & $\begin{array}{c}\text { Value of } \\
\psi=\end{array}$ & $\begin{array}{c}\text { Curviness, } \\
+ \text { or }-\end{array}$ & $\begin{array}{c}\text { Which } \\
\text { circle \#? }\end{array}$ & $\begin{array}{c}\text { Steering wheel } \\
\text { sketch }\end{array}$ \\
\hline 0.5 & & & & & \\
\hline 1 & & & & & \\
\hline 1.5 & & & & & \\
\hline
\end{tabular}

FIG. 5. A typical table for different kinds of curves in the curviness activity. Students had to fill in this table (for these and more values) for linked semicircles (Fig. 4), sinusoidal, and exponential curves.

patterns that might not be obvious if they were to answer the questions one by one. The curviness activity, in particular, had many different tables to fill in. We wanted students to use the tables to see things that were independent of each other and other things that were clearly dependent on each other. To illustrate the kinds of tables students had to fill out, we include an example of questions we asked of Ed's semicircles (as seen in Fig. 4) as well as sinusoidal and exponential functions (Fig. 5).

We note that some of these entries made sense only in the context of the activity. The "circle \#" referred to the activity described in the previous paragraph (smaller circles had higher numbers and a higher curviness), and a student's sketch of the steering wheel should be consistent with the circle \#. We also note that the sign of the curviness ( + or - ) is what we refer to as concavity in a mathematics class.

\section{Cuing one form of existing knowledge over another}

At other times, we asked questions which highlighted differences of importance. In Fig. 6, we asked students to define properties of what we called an "s function," a name we gave to a sinusoidal curve. We had several reasons for not using "sine curve" at this moment. First was the classic admonition of "idea first, name later" [36]. Second, we were aware that students at this level had all had some experience with sines, cosines, and tangents in previous instruction (either in science or in math), but that they remembered at times very little of it. We were not seeking to build on a definition of sine as opposite over hypotenuse in a unit circle. We were not interested in sines as solutions to certain differential equations, either. We were interested in the shapes that were observed, and a graph that snakes back and forth across the axis (when driving from left to right) is intuitively called an $s$ curve. We show an example as part of Fig. 6.

The questions in Fig. 6 were designed to both define $s$ functions and bring in a needed level of precision in their definition. In question 1, students were asked for more precision about the way the steering wheel, for example, might be turned at different locations on the curve. In question 2, they were asked to connect their answers here to patterns observed in a table on the previous page. In question 3 , the $s$ function was defined, and students were asked to comment on a simple (but incomplete) definition. If they did not recognize what was missing from the student quote, we expected them to recognize the differences by question 5. In question 4, we asked them to tell a version of the story we gave in Sec. IV B 1.

We avoided using the term sinusoidal until students had worked through some ideas about $s$ functions, and we similarly avoided using the term exponential until students had worked through similar ideas about $e$ functions. This may seem inconsistent with the design principle of building on existing student knowledge, but for our purposes, it was a question of focus rather than avoidance. Students, we believed, had extensive knowledge with graphing and also with sine, cosine, and tangent. We wanted them to use their graphing knowledge rather than the knowledge of the unit

Compare the curviness of the function shown below to the value of the function. (The value of the function at $t=7$ seconds, for example, is approximately $0.6 \mathrm{~cm}$ )

1. When the value of the function is positive, is the curviness positive or negative? Which parts of section $B$ on the previous page support your answer?

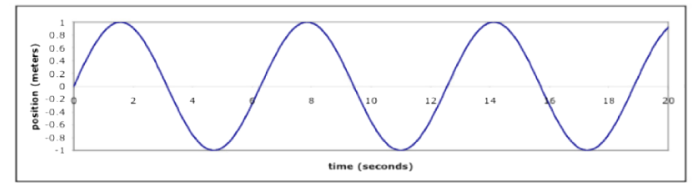

2. When the value of the function is negative, is the curviness positive or negative? Again, support your answers with examples you looked at in part B.

3. We will call a function that has the behavior described in this section an s-function. A student in this class says to your group, "s-functions always curve towards the horizontal axis". Comment on whether or not this is an appropriate description of s-functions.

4. Imagine driving down an s-function and tell the story of your steering wheel, using words and steering wheel pictures.

5. How, if at all, is this different from the story of Ed's steering wheel as he drives through the pylons?

FIG. 6. Excerpt 2 from the curviness tutorial. Students develop the idea of a sinusoidal function using language that focuses on specific ideas, and compare this situation to the one described in Fig. 4. 
circle. In the case of exponentials, we wanted to avoid any confusions they might have between powers of $x$ (such as $x^{2}$ ) and exponentials of $x\left(e^{x}\right)$. By choosing an uncommon name, we tried to isolate the learning situation briefly from their existing knowledge and tried to give them the opportunity to develop new ideas without being cued to think about unproductive ideas along the way.

\section{Stitching together functions}

One key aspect of the course was the metaphor of stitching together ideas. This was an extension of an idea introduced early in the course when discussing superposition of waves. In that case, we had applied superposition to scientific problem solving. One could break up a complicated problem into many smaller parts, address each part individually, and then "add up" the solutions (like with superposition) into a single solution to the overarching problem. This idea was used and repeatedly discussed in class. We note that the student response to the "both and or choose one" question, in Sec. IVA 3, was an example of superposition, in that the student used both ideas to give a complete answer to the problem.

When discussing driving down the wave function, we extended the superposition metaphor to include information about how one put together each of the individual solutions. We returned to the metaphor of driving (see Fig. 7).

The graphical Schrödinger equation, together with its rules about what shapes were allowed to be drawn, was the model that guided our exploration of quantum physics topics. The idea of stitching smoothly (continuity) was combined with the ideas of a finite area under the curve (normalizability) to help determine which wave functions were allowed for a given physical system whose potential energy diagram we could draw. In the follow-up labs to the curviness activity, students developed these ideas and connected them to probability density. They used PhysLets [37] to find that only certain energies led to curves which satisfied all the rules we had defined in class.

In contrast to a typical physics class, where the infinite square well is the easiest to teach because of the boundary conditions that the wave function be zero at the edge, leaving a simple differential equation to solve analytically inside the well, we found that the infinite square well was a serious challenge to students. In a finite square well, there are clear regions of exponential and sinusoidal functions. When the function has to be zero at negative and positive infinity in order to have a finite area under the curve, it is easy to stitch together a function that "leaves the highway," curves back toward the axis as an $s$ function would, and "merges back onto the highway," in order to describe the ground state energy. For an infinite square well, students needed to think of an infinite curviness at the boundary between the well and the area outside the well. This was done by considering the situation as a limiting case when $\mathrm{TE}_{\text {particle }}-\mathrm{PE}_{\text {system }}$ gives an infinite curviness as $\mathrm{PE}_{\text {system }}$ gets larger and goes toward infinity. In this case, infinite

Imagine that you are a road contractor, and you have to connect the small sections of roads into one long road for a subdivision. The cheap way to connect road segments is using short, straight lines. More expensive, but easier to drive on, is to connect each segment smoothly, with no kinks. Connect the following road sections the cheap way and the expensive way.

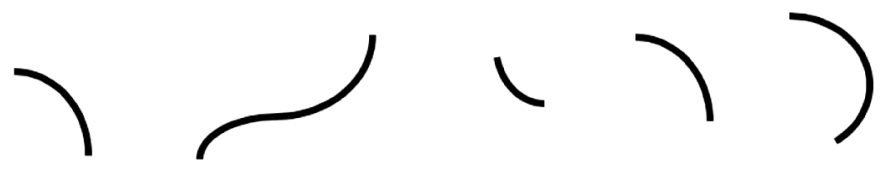

When two functions connect, they have to connect smoothly without any kinks. When two functions are properly connected, we say they are stitched together. Stitch together the two functions to the right. Does the segment you drew go higher than the left edge of the function on the right? Why or why not?

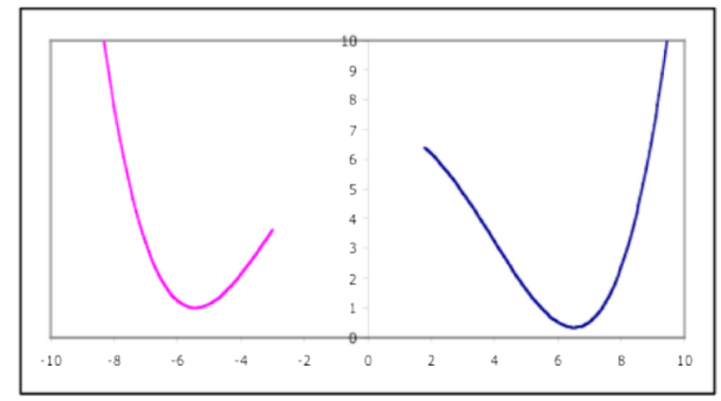

We created the above function by stitching together several s-functions and e-functions. On the graph above, identify where the function behaves like an s-function, and where the function behaves like an e-function. Explain your reasoning.

FIG. 7. Stitching together wave functions. Students consider how to connect functions and also consider whether these functions resemble sinusoidal or exponential functions. 
curviness was considered to be a kink in the curve. Students struggled with this concept when we first taught it, and we stopped teaching it. Because the infinite square well is an example chosen because of its analytical solvability, and this was not a criterion of concern in our course, we felt confident that sticking to the finite square well was sufficient for our purposes.

For example, when placing two finite square wells close to each other (an example from unit 3 of the course), students could model the ground state energy of the system and show that it was lower than the ground state energy of a single finite square well. In this way, they were able to apply their ideas of stitching to ever more complicated situations and interpret the model they had developed to understanding ever more complex situations in our physical world.

\section{Problems with epistemology}

We note that the entire second unit was problematic to us, with regard to personal epistemology. This was a very didactic unit, in that students were being told what they had to learn and how to learn it. Though the topics of probability and energy had been motivated by observations of wave-particle duality, the instruction that followed did not seem to grow naturally out of what had come previously. Where unit 1 had ended with scores suggesting better attitudes about the coherence of science (as measured by the MPEX [10,29]), we noticed that these attitudes changed during unit 2. To the answer of "how do you know?" a perfectly reasonable and appropriate answer would be "because you told me." It was also perfectly reasonable that students not really believe us on the relevance of some of the content. Though we discussed the idea in class, it was not necessarily helpful to tell students that we were building a model with enormous explanatory power and that the model itself was merely an example of the act of modeling, as a whole.

To address these concerns, we tried to emphasize those moments where the content connected to things that students already knew. When discussing energy, we used macroscopic objects, and tried to help students understand the differences between the system and the objects in the system. When discussing probability density, we used the example of a ball tossed in the air. Students had intuitions about the speed of the ball at different heights, and we used these intuitions to guide the instruction [22]. Similarly, classroom discussions of time spent in locations involved questions like "how likely is it that you're in class on a given day?" These were our attempts to have students think of everyday events in the context of what we were learning in class, and to use their understanding of everyday events to guide their thinking about the physics. When discussing the curviness of a wave function, we asked students to consider how to drive down a function, as if the graphical representation of the wave function was a map of the road on which they were driving. When interpreting the various rules of normalizability and continuity, we turned those into rules about driving (leaving the highway and returning to it, and no kinks in our curves).

Still, we were not satisfied with this section of the course in terms of promoting productive personal epistemologies. We had successfully used graphical tools to solve complicated differential equations with difficult boundary conditions, and had used everyday intuitions and examples to help with reasoning through these situations. We had given students ample time (several weeks) to develop these ideas, but recognized that they still struggled with understanding why the rules we gave them were the rules they had to use. Our design principles had stated that we could use quantum physics as a platform to advance one's personal epistemology regarding science, and here we were, proscribing what was to be learned to the students.

Only in unit 3 did we try to have the pieces come together. The goal was to explore, slowly and carefully, what a model could do. We used the graphical Schrödinger equation throughout this unit. Atoms were modeled as onedimensional finite square wells. Students used Physlets [37] to guess energy values and see if their guesses led to allowable wave functions. From this, they determined that only certain energies were allowed, meaning that energy quantization arose because only some energies led to graphs that satisfied the rules learned at the end of unit 2. Subsequent discussion of using light to add energy to (or remove energy from) an atom let us talk about spectroscopy. The same arguments about wave function curviness were used to understand simple models of a molecule of two atoms (finite square wells) sharing a single electron. The ideas were also applied to quantum tunneling. Students used classical analogues to model the barrier and discussed differences between classical and quantum situations (in that there is a probability of finding particles in classically forbidden regions). Evidence suggested that they learned these ideas well [38-40].

Though the third unit was less didactic than unit 2, the course material was not necessarily relevant to students. The content of the physics was also not the core point of instruction. Instead, the course emphasized issues of epistemology related to the quantum physics. To the question of "how do you know?" an answer might then be that the student figured something out, using the toolbox they had been given. To the question of "why do I believe this result?" an answer might be that the observed phenomenon was consistent with the model that we were using. If our model was good enough to find discrete energy levels, and light was given off or absorbed during transitions between energy levels, then we could assume that certain frequencies of light could characterize certain atoms. We discussed the discovery of helium in the sun from this perspective, for example. Finally, to the question of "How can I explain that?" an answer might be that we 
had collectively built a model that allowed us to describe such diverse situations as atomic spectra, molecular bonding, and quantum tunneling.

\section{DISCUSSION}

In the previous section, we described in detail two examples of activities in which essential elements of our course can be observed in the materials that students worked with. These two activities were consistent with our overall design principles. First, we used quantum physics as a way of studying deeper issues of personal epistemology, even when situations like the overall didactic nature of unit 2 weakened our overall message. Second, rather than emphasize mathematics as is so often done in a typical quantum physics course for physics majors, we used a graphical method. To do so, we tapped into student knowledge of everyday events like driving. Once students had used the idea of driving down functions to help solve the graphical Schrödinger equation (3), we applied several rules to create a model that led to quantization. We used everyday events in other aspects of the course, as well, such as when considering the differences between situations where you could do two things at once or you could only do one thing or the other. This distinction, common to everyday social lives, helped us make sense of the dilemmas of wave-particle duality. Finally, we allowed these ideas to be developed primarily in a lab setting where students worked primarily in small groups at their own pace, though this was modified over time to help students take more ownership of their learning through the use of whiteboard meetings at regular intervals in the course.

Through these activities, we were able to strengthen students' personal epistemologies in the context of science, for example by emphasizing the coherence of the physics, build a model that was difficult but had great explanatory power, and address contradictions that might seem otherwise impossible to address. These were the core outcomes that we sought in the course especially when students were taking possibly their last science course.

Importantly, certain metaphors were used throughout the course. One core metaphor was the idea of superposition. The idea was introduced in wave physics, where the effects of multiple waves add up at a single point if those waves are at that point at the same time. Without the idea of superposition, the conceptual dilemmas of wave-particle duality could not be defined. We applied the idea to the course itself, as well. So, when solving the curviness equation in different regions of space, students had to "stitch together" the different solutions using the provided rules (e.g., no kinks in the curve.). As students learned to interpret the equation and the wave functions that satisfied a given situation, students had to stitch new concepts together, as well. Much like in superposition of waves, each idea could be taught individually, but the effects of all the ideas together led to what was observed. This idea of stitching together separately developed ideas into a single, coherent story was the overarching conceptual framework for our course. In being explicit about this as our goal, we hoped to help students understand the process of using models to provide rich descriptions and possibly also predictions of the world around us.

Students' ways of reasoning about the physics were at least as important as the physics content. By the end of the course, we hoped that students could say "I don't know if I believe this, but I can think about it and arrive at an answer that matches things we can observe." Further, what we meant by "I can think about it" was that they could split a problem into its individual parts, think about each situation separately and independently, and then stitch together their thinking in a way that built a larger, more complex analysis than when analyzing a single simple situation, individually. For general education students who were possibly taking their last science class, the idea of being able to work with a model and explore its possibilities was more important to us than the content of quantum physics itself.

\section{CONCLUSION}

In this paper, we have described the principles we used when developing the Intuitive Quantum Physics course. We first presented the principles in general form, then described how they influenced the design of the course as a whole, and then described how they influenced the design of individual activities, down to the level of individual questions. In laying out this detail, our goal has been to be clear in how design principles can influence multiple levels of course design. By illustrating these design decisions, we hope to make clear one approach by which curriculum development can lead to principled instruction that helps students develop the multiple skills that we, as physics instructors, have for our students.

\section{ACKNOWLEDGMENTS}

We thank the National Science Foundation, DUE0410895, for their support in developing this course. We also thank Katrina Black, David Clark, Roger Feeley, and Eleanor Sayre for their substantial contributions in developing the Intuitive Quantum Physics course, as well as the students who took it and from whom we learned as we redesigned the course to better meet their needs. 
[1] E. F. Redish and R. N. Steinberg, A New Model Course in Quantum Mechanics for Scientists and Engineers (University of Maryland PERG, College Park, MD, 2002), https://www.physics.umd.edu/perg/qm/qmcourse/ NewModel/qmtuts.htm.

[2] M. C. Wittmann, R. N. Steinberg, and E. F. Redish, Activity-Based Tutorials Volume 2: Modern Physics (John Wiley \& Sons, Inc., New York, 2005).

[3] M. C. Wittmann, The object coordination class applied to wave pulses: Analysing student reasoning in wave physics, Int. J. Sci. Educ. 24, 97 (2002).

[4] M. C. Wittmann, Making Sense of How Students Come to an Understanding of Physics: An Example from Mechanical Waves (University of Maryland, College Park, MD, 1998).

[5] B. S. Ambrose, P. S. Shaffer, R. N. Steinberg, and L. C. McDermott, An investigation of student understanding of single-slit diffraction and double-slit interference, Am. J. Phys. 67, 146 (1999).

[6] C. J. Linder, University physics students' conceptualizations of factors affecting the speed of sound propagation, Int. J. Sci. Educ. 15, 655 (1993).

[7] M. C. Wittmann, R. N. Steinberg, and E. F. Redish, Investigating student understanding of quantum physics: Spontaneous models of conductivity, Am. J. Phys. 70, 218 (2002).

[8] R. Müller and H. Wiesner, Teaching quantum mechanics on an introductory level, Am. J. Phys. 70, 200 (2002).

[9] L. Bao and E. F. Redish, Understanding probabilistic interpretations of physics systems: A prerequisite to learning quantum physics, Am. J. Phys. 70, 210 (2002).

[10] E. F. Redish, J. M. Saul, and R. N. Steinberg, Student expectations in introductory physics, Am. J. Phys. 66, 212 (1998).

[11] D. Hammer and A. Elby, On the form of a personal epistemology, in Personal Epistemology: The Psychology of Beliefs about Knowledge and Knowing, edited by B. K. Hofer and P. R. Pintrich (Lawrence Erlbaum Associates, Mahwah, NJ, 2002), pp. 169-190.

[12] J. P. Smith, A. A. diSessa, and J. Roschelle, Misconceptions reconceived: A constructivist analysis of knowledge in transition, J. Learn. Sci. 3, 115 (1993).

[13] E. Mazur, Peer Instruction: A User's Manual (Prentice Hall, Upper Saddle River, NJ, 1997).

[14] E. Brewe, L. Kramer, and G. O'Brien, Modeling instruction: Positive attitudinal shifts in introductory physics measured with CLASS, Phys. Rev. ST Phys. Educ. Res. 5, 013102 (2009).

[15] M. C. Wittmann, R. N. Steinberg, and E. F. Redish, Activity-Based Tutorials Volume 1: Introductory Physics (John Wiley \& Sons, Inc., New York, 2004).

[16] E. F. Redish, Teaching Physics: With the Physics Suite (John Wiley \& Sons, Inc., New York, 2003).

[17] L. C. McDermott and P. S. Shaffer, The Physics Education Group at the University of Washington, Instructor's Guide for Tutorials in Introductory Physics (Prentice Hall, Upper Saddle River, NJ, 2003).

[18] M. C. Wittmann, R. N. Steinberg, and E. F. Redish, Making sense of how students make sense of mechanical waves, Phys. Teach. 37, 15 (1999).
[19] K. Wosilait, P. R. L. Heron, P. S. Shaffer, and L. C. McDermott, Addressing student difficulties in applying a wave model to the interference and diffraction of light, Am. J. Phys. 67, S5 (1999).

[20] S. Vokos, P. S. Shaffer, B. S. Ambrose, and L. C. McDermott, Student understanding of the wave nature of matter: Diffraction and interference of particles, Am. J. Phys. 68, S42 (2000).

[21] R. N. Steinberg, M. C. Wittmann, L. Bao, and E. F. Redish, The influence of student understanding of classical physics when learning quantum mechanics, in Proceedings of the NARST Annual Meeting, edited by D. Zollman (Boston, MA 1999).

[22] M. C. Wittmann, J. T. Morgan, and R. Feeley, Laboratorytutorial activities for teaching probability, Phys. Rev. ST Phys. Educ. Res. 2, 020104 (2006).

[23] E. F. Redish, D. Hammer, and A. Elby, Learning How to Learn Science: Physics for bioscience Majors (University of Maryland PERG, College Park, MD, 2000), https:// www.physics.umd.edu/ripe/perg/role/ROLEProp.pdf.

[24] A. Elby and D. Hammer, On the substance of a sophisticated epistemology, Sci. Educ. 85, 554 (2001).

[25] D. Hammer and A. Elby, Tapping epistemological resources for learning physics, J. Learn. Sci. 12, 53 (2003).

[26] L. Lising and A. Elby, The impact of epistemology on learning: A case study from introductory physics, Am. J. Phys. 73, 372 (2005).

[27] R. E. Scherr and A. Elby, Enabling informed adaptation: Open-source physics worksheets integrated with implementation resources, AIP Conf. Proc. 883, 46 (2006).

[28] A. Elby and R. E. Scherr, Open source tutorials, PhysPort. Org., 07/05/2019, https://www.physport.org/methods/ method.cfm?G=OST_Tutorials (2006).

[29] T. McCaskey, Comparing and contrasting different methods for probing student epistemology and epistemological development in introductory physics (University of Maryland, College Park, MD, 2009), https://drum.lib.umd.edu/handle/ 1903/9824.

[30] P. G. Merli, G. F. Missiroli, and G. Pozzi, On the statistical aspect of electron interference phenomena, Am. J. Phys. 44, 306 (1976).

[31] P. A. Tipler, Modern Physics (W.H. Freeman and Company, New York, 1977), ISBN 13: 9781429250788 ISBN: $142925078 X$.

[32] D. Hammer, Discovery learning and discovery teaching, Cognit. Instr. 15, 485 (1997).

[33] D. Hammer and D. Schifter, Practices of inquiry in teaching and research, Cognit. Instr. 19, 441 (2001).

[34] P. Jolly, D. Zollman, N. S. Rebello, and A. Dimotrova, Visualizing motion in potential wells, Am. J. Phys. 66, 57 (1998).

[35] A. P. French and E. F. Taylor, An Introduction to Quantum Physics (W.W. Norton \& Company Inc., New York, 1978).

[36] A. B. Arons, A Guide to Introductory Physics Teaching (John Wiley \& Sons Inc., New York, 1990). 
[37] W. Christian and M. Belloni, Physlets: Teaching Physics with Interactive Curricular Material (Prentice Hall, Upper Saddle River, NJ, 2001).

[38] J. T. Morgan, M. C. Wittmann, and J. R. Thompson, Student understanding of tunneling in quantum mechanics: examining interview and survey results for clues to student reasoning, AIP Conf. Proc. 720, 97 (2004).
[39] J. T. Morgan, Examining the evolution of student ideas about quantum tunneling, AIP Conf. Proc. 818, 73 (2006).

[40] M. C. Wittmann, J. T. Morgan, and L. Bao, Addressing student models of energy loss in quantum tunneling, Eur. J. Phys. 26, 939 (2005). 